\title{
Light dark matter in the NMSSM: upper bounds on direct detection cross sections
}

\section{Debottam Das and Ulrich Ellwanger}

Laboratoire de Physique Théorique, UMR 862\%, CNRS and Université de Paris-Sud, Bât. 210, 91405 Orsay, France

E-mail: ulrich.ellwanger@th.u-psud.fr, debottam.das@th.u-psud.fr

Abstract: In the Next-to-Minimal Supersymmetric Standard Model, a bino-like LSP can be as light as a few $\mathrm{GeV}$ and satisfy WMAP constraints on the dark matter relic density in the presence of a light CP-odd Higgs scalar. We study upper bounds on the direct detection cross sections for such a light LSP in the mass range $2-20 \mathrm{GeV}$ in the NMSSM, respecting all constraints from B-physics and LEP. The OPAL constraints on $e^{+} e^{-} \rightarrow \chi_{1}^{0} \chi_{i}^{0}$ $(i>1)$ play an important rôle and are discussed in some detail. The resulting upper bounds on the spin-independent and spin-dependent nucleon cross sections are $\sim 10^{-42} \mathrm{~cm}^{2}$ and $\sim 4 \times 10^{-40} \mathrm{~cm}^{2}$, respectively. Hence the upper bound on the spin-independent cross section is below the DAMA and CoGeNT regions, but could be compatible with the two events observed by CDMS-II.

KEYWORDS: Supersymmetry Phenomenology

ARXIV EPRINT: 1007.1151 


\section{Contents}

1 Introduction 1

2 The NMSSM and the impact of its parameters on the LSP cross sections 3

3 Experimental constraints on the parameter space $\quad 6$

3.1 Constraints from sparticle and Higgs searches 6

3.2 Constraints from B-physics 8

$\begin{array}{lll}3.3 & \text { Additional constraints } & 9\end{array}$

$4 \quad$ Results and conclusions $\quad 9$

\section{Introduction}

The DAMA [1] and CoGeNT [2] dark matter detection experiments have reported events in excess of the expected background, which would be compatible with a WIMP mass of a few GeV. Also the CDMS-II experiment [3] has reported two events, which could be explained by a WIMP mass of $\gtrsim 10 \mathrm{GeV}$ (or background). On the other hand, exclusion limits from the CRESST-I [4], Xenon10 [5], TEXONO [6], Xenon100 [7] and CDMS-Si [8] experiments set upper bounds on the spin-independent detection cross sections for this mass range of a WIMP, some of which seem incompatible with the reported hints for a signal.

In any case, it is important to know whether specific models for dark matter with a WIMP mass of a few $\mathrm{GeV}$ can produce direct detection cross sections compatible with the reported excesses, and/or whether regions in the parameter space of such models can be tested by present and future exclusion limits.

Supersymmetric (Susy) extensions of the Standard Model are popular, amongst others, since they predict naturally (for unbroken R-parity, and for a neutral Lightest Supersymmetric Particle (LSP)) a candidate for dark matter, with a relic density compatible with WMAP constraints [9]. Within the Minimal Supersymmetric extension of the Standard Model (MSSM) four neutral fermions (neutralinos $\chi_{i}^{0}, i=1 \ldots 4$ ) exist, which are composed of the bino (superpartner of the $\mathrm{U}(1)_{Y}$ gauge boson), the wino (superpartner of the $W_{\mu}^{3}$ gauge boson) and two higgsinos (superpartners of neutral Higgs bosons). These states mix, and the lightest neutralino $\chi_{1}^{0}$, which is the lightest eigenvalue of the $4 \times 4$ mass matrix, will be the LSP (leaving aside the possibility of a sneutrino LSP).

Often the LSP is dominantly bino-like, whose mass $m_{\chi_{1}^{0}}$ is approximately given by the soft Susy breaking gaugino mass $\sim M_{1}$. Assuming unification of the three gaugino masses for the bino $\left(M_{1}\right)$, the winos $\left(M_{2}\right)$ and the gluino $\left(M_{3}\right)$ at the scale of Grand Unification, $M_{1}$ is naturally the smallest among these mass terms at the electroweak scale. However, due to the lower bound of $\sim 100 \mathrm{GeV}$ on $M_{2}$ from the lower bound on chargino masses, the 
assumption of unification of the three gaugino masses implies $M_{1} \gtrsim 50 \mathrm{GeV}$ and a similar lower bound on the mass of the LSP.

The assumption of unification of the three gaugino masses can be dropped, however, in that case $M_{1}$ and hence the LSP mass $m_{\chi_{1}^{0}}$ can be arbitrarily small. Then, on the other hand it can become difficult to satisfy the WMAP constraint on the dark matter relic density i.e., to reduce the dark matter relic density after the Big Bang to an acceptable value compatible with this constraint. To this end, dark matter annihilation processes have to be sufficiently effective. The following pair annihilation processes can be relevant: exchange of Susy partners of fermions (sfermions, in particular sleptons) in the t-channel, and Z-exchange or Higgs-exchange in the s-channel (if the LSP has a sufficiently large higgsino component). In addition, neutralinos can co-annihilate with other sparticles if they have similar masses, but co-annihilation processes will not be relevant for a light LSP as considered here. In the MSSM, sufficiently effective dark matter annihilation processes impose constraints on a light LSP:

Considering LSP annihilation via slepton exchange in the t-channel, a lower bound $m_{\chi_{1}^{0}} \gtrsim 18 \mathrm{GeV}$ was derived in $[10,11]$ from the lower bound of $\sim 100 \mathrm{GeV}$ on the slepton masses. (Relaxing this bound to $\gtrsim 80 \mathrm{GeV}$ for stau masses, one obtains $m_{\chi_{1}^{0}} \gtrsim 13 \mathrm{GeV}[10-12]$, unless the LSP mass is very small corresponding to hot dark matter [12].) Allowing for LSP annihilation via CP-odd Higgs $(A)$ exchange in the s-channel, a lower limit $m_{\chi_{1}^{0}} \gtrsim 6 \mathrm{GeV}$ was given in [13-16] from $m_{A} \gtrsim 90 \mathrm{GeV}$ for large values of $\tan \beta \gtrsim 25$. However, as noted in [17], this region of the parameter space of the MSSM is now strongly constrained by the bounds on $B_{s} \rightarrow \mu^{+} \mu^{-}$. A LSP with a mass in the $5-15 \mathrm{GeV}$ range in the MSSM has been considered in [18] without, however, asking for a correct relic density.

In the Next-to-Minimal Supersymmetric Standard Model (NMSSM, see [19, 20] for recent reviews), which can solve the $\mu$-problem of the MSSM, the Higgs and neutralino sectors are extended by gauge singlet states. As noticed in [21-24], the mass of the LSP can be considerably smaller in the NMSSM than in the MSSM and can still be compatible with the WMAP constraint on the relic density. This is a consequence of a light CP-odd Higgs boson in the spectrum (on top of the CP-odd Higgs boson of the MSSM), which can be mostly singlet-like and which is not ruled out by LEP-constraints. Then, sufficiently large LSP annihilation cross sections via the exchange of this additional CP-odd Higgs boson in the s-channel may be possible even for a light LSP with mass of a few GeV.

A light LSP in the NMSSM could be a (dominantly) singlet-like state; in this case, however, its direct detection cross sections would be tiny. On the other hand, as in the MSSM, a light LSP in the NMSSM can originate from a small value of $M_{1}$ in which case it will be dominantly bino-like and can have larger direct detection cross sections. These have been estimated in [23], where also constraints on the corresponding parameter space from B-physics, LEP and $\Upsilon$-physics were discussed. However, the corresponding points in the parameter space given as examples in [23] suffer from a negative effective $\mu$-parameter (which is in conflict with the measured anomalous magnetic moment of the muon), and not all experimental constraints considered below are taken into account.

In view of the interest in a light LSP with a mass in the $2-20 \mathrm{GeV}$ range, we find it 
appropriate to study upper bounds on its direct detection cross sections in the NMSSM. Direct detection cross sections in the NMSSM including WMAP constraints have been studied before in [25-31], but not for the LSP mass range considered here. Apart from WMAP constraints, we take care of a lengthy list of experimental constraints from Bphysics (important for large $\tan \beta$ and/or relatively light charged and CP-odd Higgs bosons as relevant here), $\Upsilon$-physics and LEP-constraints on neutralino production. Among the latter, OPAL limits on $e^{+} e^{-} \rightarrow \chi_{1}^{0} \chi_{i}^{0}(i>1)$ turn out to be very important. Since these are also relevant for the MSSM, but have hardly been discussed before (a notable exception is [12]), we study their consequences in some detail. For the numerical analysis we use the code NMSSMTools [32, 33] coupled to micrOMEGAs [34-36]. As a result we obtain upper bounds on the spin-independent and spin-dependent LSP-nucleon cross sections of $\sigma^{\mathrm{SI}} \lesssim 10^{-42} \mathrm{~cm}^{-2}$ and $\sigma^{\mathrm{SD}} \lesssim 4 \times 10^{-40} \mathrm{~cm}^{-2}$, varying somewhat with the LSP mass in the $2-20 \mathrm{GeV}$ range. The maximal value for $\sigma^{\mathrm{SI}}$ is indeed near the estimate given in [23].

In the next section 2 we present the relevant parameters of the NMSSM and their impact on the LSP cross sections. In section 3, we discuss the relevant experimental constraints. The consequences of the OPAL constraints on $e^{+} e^{-} \rightarrow \chi_{1}^{0} \chi_{i}^{0}(i>1)$ on the parameter space (implying a lower bound on $\mu_{\text {eff }}$ ) are estimated in an analytic approximation, which reproduces well the full numerical results. Section 4 is devoted to our results and conclusions.

\section{The NMSSM and the impact of its parameters on the LSP cross sec- tions}

In the NMSSM the $\mu$ parameter of the MSSM is replaced by a Yukawa coupling $\lambda$ to a gauge singlet (super-) field $S$. Then, the vacuum expectation value (vev) $s$ of the real scalar component of $S$ generates an effective $\mu$-term

$$
\mu_{\mathrm{eff}}=\lambda s
$$

Most of the time one studies the NMSSM with a scale invariant superpotential $W$ which contains, apart from the Yukawa coupling of $S$ to the MSSM-like Higgs doublet fields $H_{u}$ and $H_{d}$, a trilinear term $\sim \frac{\kappa}{3} S^{3}$. Hence the Higgs mass term $\mu H_{u} H_{d}$ in $W_{\mathrm{MSSM}}$ is replaced by

$$
W_{\mathrm{NMSSM}}=\lambda S H_{u} H_{d}+\frac{\kappa}{3} S^{3}+\ldots .
$$

(Occasionally one considers the so-called nMSSM [24, 27, 30] without the trilinear coupling $\sim \frac{\kappa}{3} S^{3}$, which is replaced by a tadpole-term $\sim \xi_{F} S$.) Compared to the MSSM, the gauge singlet superfield $S$ adds additional degrees of freedom to the CP-even and CP-odd Higgs sectors as well as to the neutralino sector. Hence the spectrum contains

- 3 CP-even neutral Higgs bosons $H_{i}, i=1,2,3$, which mix in general;

- 2 CP-odd neutral Higgs bosons $A_{1}$ and $A_{2}$;

- one charged Higgs boson $H^{ \pm}$; 
- five neutralinos $\chi_{i}^{0}, i=1 \ldots 5$, which are mixtures of the bino, the neutral wino, the neutral higgsinos and the singlino;

- two charginos which are mixtures of the charged winos and the charged higgsinos.

Apart from the Susy generalisations of the Standard-Model-like gauge and Yukawa couplings and the superpotential in eq. (2.2), the Lagrangian of the NMSSM contains soft Susy breaking terms in the form of gaugino masses $M_{1}, M_{2}$ and $M_{3}$ for the bino, the winos and the gluino, respectively, mass terms for all scalars (squarks, sleptons, Higgs bosons including the singlet $S$ ) as well as trilinear scalar self-couplings as $\lambda A_{\lambda} S H_{u} H_{d}+\frac{\kappa}{3} A_{\kappa} S^{3}$, which reflect the trilinear couplings among the superfields in the superpotential.

Expressions for the mass matrices for all Higgs- and neutralino states can be found in $[19,20]$; below we confine ourselves to those which are of relevance subsequently. Dropping the Goldstone mode, the $2 \times 2$ mass matrix for the CP-odd Higgs bosons $\mathcal{M}_{P}^{2}$ in the basis $\left(A_{\mathrm{MSSM}}, S_{I}\right)$ has the elements

$$
\begin{aligned}
& \mathcal{M}_{P, 11}^{2}=\frac{2 \mu_{\mathrm{eff}}\left(A_{\lambda}+\kappa s\right)}{\sin 2 \beta} \equiv M_{A}^{2}, \\
& \mathcal{M}_{P, 22}^{2}=\lambda\left(A_{\lambda} / s+4 \kappa\right) v_{u} v_{d}-3 \kappa A_{\kappa} s \\
& \mathcal{M}_{P, 12}^{2}=\lambda\left(A_{\lambda}-2 \kappa s\right) v
\end{aligned}
$$

where $v_{u}, v_{d}$ denote the vevs of $H_{u}, H_{d}$, respectively, $v=\sqrt{v_{u}^{2}+v_{d}^{2}} \sim 174 \mathrm{GeV}$ and, as usual, $\tan \beta=v_{u} / v_{d}$. The matrix element $\mathcal{M}_{P, 11}^{2}$ would be the mass squared of the MSSMlike CP-odd scalar $A_{\mathrm{MSSM}}$, if the singlet sector were absent; subsequently we will denote it simply by $M_{A}^{2}$. (This parameter can replace the parameter $A_{\lambda}$.) For any (possibly large) value of $M_{A}^{2}, \mathcal{M}_{P}^{2}$ can have another small eigenvalue corresponding to an additional light CP-odd Higgs boson $A_{1}$ which is mostly singlet-like. This state will be relevant for the LSP annihilation cross section below.

The mass of the charged Higgs scalar is given by

$$
M_{H^{ \pm}}^{2}=M_{A}^{2}+v^{2}\left(\frac{g_{2}^{2}}{2}-\lambda^{2}\right)
$$

note that it decreases with increasing $\lambda$. As is well known, too small values of $M_{H^{ \pm}}$can cause disagreements between measurements and corresponding contributions to B-physicsobservables as $b \rightarrow s \gamma$; this will be of importance below.

Notably for large $M_{A}$, one of the 3 CP-even Higgs bosons will have a mass close to $M_{A}$. In the MSSM, the corresponding CP-even state is denoted by $H$, and we will maintain this denomination. The spin-independent LSP-nucleon cross section will be dominated by the exchange of this CP-even scalar $H$, since its couplings to down-type quarks (particularly the strange quark) are enhanced for large values of $\tan \beta$.

Also, the mass of the charged Higgs scalar is close to $M_{A}$ for large $M_{A}$; then the states $H, A_{\mathrm{MSSM}}$ and $H^{ \pm}$form a nearly degenerate $\mathrm{SU}(2)$ doublet. In fact this approximate degeneracy holds down to fairly low values of $M_{A} \sim 300 \mathrm{GeV}$. 
In the neutralino sector, the bino $\lambda_{1}$ and the neutral wino $\lambda_{2}^{3}$ mix with the neutral higgsinos $\psi_{d}^{0}, \psi_{u}^{0}$ and the singlino $\psi_{S}$, and generate a symmetric $5 \times 5$ mass matrix $\mathcal{M}_{0}$. In the basis $\psi^{0}=\left(-i \lambda_{1},-i \lambda_{2}^{3}, \psi_{d}^{0}, \psi_{u}^{0}, \psi_{S}\right), \mathcal{M}_{0}$ reads

$$
\mathcal{M}_{0}=\left(\begin{array}{ccccc}
M_{1} & 0 & -\frac{g_{1} v_{d}}{\sqrt{2}} & \frac{g_{1} v_{u}}{\sqrt{2}} & 0 \\
& M_{2} & \frac{g_{2} v_{d}}{\sqrt{2}} & -\frac{g_{2} v_{u}}{\sqrt{2}} & 0 \\
& & 0 & -\mu_{\mathrm{eff}} & -\lambda v_{u} \\
& & & 0 & -\lambda v_{d} \\
& & & & 2 \kappa s
\end{array}\right) .
$$

It can be diagonalized by an orthogonal real matrix $N_{i j}$ such that the physical masses $m_{\chi_{i}^{0}}$ ordered in $\left|m_{\chi_{i}^{0}}\right|$ are real (but not necessarily positive). Denoting the 5 eigenstates by $\chi_{i}^{0}$, we have

$$
\chi_{i}^{0}=N_{i j} \psi_{j}^{0} .
$$

Finally, the chargino masses are described by a $2 \times 2$ mass matrix containing $M_{2}$ and $\mu_{\text {eff }}$ as diagonal entries. The lower bound of $\sim 103 \mathrm{GeV}$ on the lightest chargino implies at least the constraint

$$
\operatorname{Min}\left\{M_{2},\left|\mu_{\mathrm{eff}}\right|\right\} \gtrsim 100 \mathrm{GeV}
$$

(one can choose $M_{2}>0$ by convention).

Next, we discuss the dominant contribution to the spin-independent LSP-nucleon cross section $\sigma^{\mathrm{SI}}$. Leaving aside scenarios with light squark masses of $\sim 100 \mathrm{GeV}$ (which are difficult to reconcile with Tevatron constraints), $\sigma^{\mathrm{SI}}$ is dominated by the exchange of CPeven Higgs bosons, which couple mostly to the strange quark sea. Among the CP-even Higgs bosons, the coupling of the state $H$ to down-type quarks (as the strange quark) increases with $\tan \beta$. Hence, although its mass is generally larger than the mass of the Standard-Model-like Higgs boson $h, H$-exchange provides the leading contribution to $\sigma^{\text {SI }}$ for large values of $\tan \beta$. Then, the dominant component of $H$ is given by $H_{d}$.

The dominant coupling of $H \sim H_{d}$ to the LSP is induced by the bino-higgsino-Higgs vertex $\sim g_{1}$ and hence proportional to $g_{1} N_{11} N_{13}$, where $N_{11}$ denotes the bino- and $N_{13}$ the $\psi_{d}^{0}$-higgsino-component of the LSP. All in all one finds

$$
\sigma^{\mathrm{SI}} \sim N_{11}^{2} N_{13}^{2} \frac{\tan ^{2} \beta}{m_{H}^{4}},
$$

which shows that the largest values of $\sigma^{\mathrm{SI}}$ are obtained for a large product $N_{11} N_{13}$, large $\tan \beta$ and low values of $m_{H}$.

The dominant contribution to the spin-dependent LSP-nucleon cross section $\sigma^{\mathrm{SD}}$ originates, as in the MSSM, from $Z$-exchange. At first sight one could imagine that, for a light CP-odd Higgs boson $A_{1}$, its exchange could also give important contributions to $\sigma^{\mathrm{SD}}$. However, the corresponding contribution vanishes in the limit of zero momentum transfer and, moreover, the coupling of its doublet component to strange quarks is always tiny compared to the $Z$-boson coupling. 
The coupling of the $Z$-boson to the LSP originates from the gauge couplings of the higgsino components $\psi_{u}^{0}$ and $\psi_{d}^{0}$. Since no additional free parameters intervene, the spindependent cross section $\sigma^{\mathrm{SD}}$ is proportional to

$$
\sigma^{\mathrm{SD}} \sim\left(N_{13}^{2}-N_{14}^{2}\right)^{2}
$$

Finally the LSP annihilation cross section $\sigma_{\text {ann }}$ is dominated, for the LSP mass range $2-20 \mathrm{GeV}$ under consideration, by the exchange of a light $A_{1}$ in the s-channel. The dominant contribution to the $A_{1} \chi_{1}^{0} \chi_{1}^{0}$ coupling is induced by the doublet component of $A_{1}$ and the bino-higgsino components of $\chi_{1}^{0}$ as in the case of the $H \chi_{1}^{0} \chi_{1}^{0}$ coupling above; the singlet components of $A_{1}$ and $\chi_{1}^{0}$ play a minor rôle here. In any case one has (neglecting the finite width of $A_{1}$ and the velocity of $\chi_{1}^{0}$ near the freeze-out temperature)

$$
\sigma_{\mathrm{ann}} \sim \frac{1}{\left(m_{A_{1}}^{2}-4 m_{\chi_{1}^{0}}^{2}\right)^{2}} .
$$

Hence $\sigma_{\text {ann }}$ can be sufficiently large for suitable values of $m_{A_{1}}^{2}$, the lightest eigenvalue of $\mathcal{M}_{P}^{2}$ in eq. (2.3). (We remark that the width of $A_{1}$ is taken into account both for the numerical calculation of $\sigma_{\text {ann }}$ and for the constraints from B-physics below.)

\section{Experimental constraints on the parameter space}

In this section we discuss various constraints on the parameters of the NMSSM, notably (but not exclusively) from LEP and B-physics, separately in various subsections.

\subsection{Constraints from sparticle and Higgs searches}

As we have seen in eq. (2.8), a large spin-independent detection cross section $\sigma^{\text {SI }}$ requires bino-components $N_{11}$ and higgsino-components $N_{13}$ of the LSP. For small $M_{1}$ such that $m_{\chi_{1}^{0}}$ is in the $2-20 \mathrm{GeV}$ range, the bino component of $\chi_{1}^{0}$ is automatically large. However, a large higgsino component of $\chi_{1}^{0}$ requires relatively small values for $\mu_{\text {eff }}$ (below $\sim 160 \mathrm{GeV}$ ) in the mass matrix $\mathcal{M}_{0}$ in eq. (2.5). Consequently the neutralino states $\chi_{2}^{0}$ and $\chi_{3}^{0}$ (for $\left.M_{2}, 2 \kappa s>\mu_{\text {eff }}\right)$ are higgsino-like with masses of the order of $\mu_{\text {eff }}$. Then, the production process $e^{+} e^{-} \rightarrow \chi_{1}^{0} \chi_{i}^{0}(i=2,3)$ was kinematically possible at LEP2, and corresponding limits from DELPHI [37] and OPAL [38] have to be taken into account.

The strongest limits come from OPAL at $208 \mathrm{GeV}$, where we can assume $100 \% Z^{*}$ branching ratios for the $\chi_{i}^{0}$ decays (see figure 10 in [38]). Upper bounds on the cross section are given in $5 \mathrm{GeV}$-wide bins of $m_{\chi_{i}^{0}}$. Since we will find $m_{\chi_{3}^{0}}-m_{\chi_{2}^{0}} \sim 40 \mathrm{GeV}$, the bounds apply for $\chi_{2}^{0}$ and $\chi_{3}^{0}$ separately. For $m_{\chi_{1}^{0}}<20 \mathrm{GeV}$, at least one of the $\chi_{2}^{0}$ or $\chi_{3}^{0}$ production cross sections (in association with $\chi_{1}^{0}$ ) is bounded from above by $0.05 \mathrm{pb}$.

In principle, both $Z^{*}$-exchange in the s-channel and selectron exchange in the t-channel contribute to this cross section. However, the interference between these channels is positive, hence the most conservative bounds on the parameters are obtained by assuming heavy selectrons and that $e^{+} e^{-} \rightarrow \chi_{1}^{0} \chi_{i}^{0}$ originates from $Z^{*}$-exchange only. The expression 
for $\sigma_{Z}\left(e^{+} e^{-} \rightarrow \chi_{1}^{0} \chi_{i}^{0}\right)$ is given, e.g., in [39] and can be written as

$$
\begin{aligned}
\sigma_{Z}\left(e^{+} e^{-} \rightarrow \chi_{1}^{0} \chi_{i}^{0}\right)= & \frac{\left(g_{1}^{2}+g_{2}^{2}\right)^{2}}{32 \pi\left(s-M_{Z}^{2}\right)^{2}}\left(N_{13} N_{i 3}-N_{14} N_{i 4}\right)^{2}\left(\frac{1}{4}-\sin ^{2} \theta_{w}+2 \sin ^{4} \theta_{w}\right) \\
& \times \frac{\sqrt{\lambda(s)}}{s}\left(E_{1} E_{i}+\frac{\lambda(s)}{12 s}-m_{\chi_{1}^{0}} m_{\chi_{i}^{0}}\right)
\end{aligned}
$$

(note the different basis for the neutralinos in [39]) with

$$
\lambda(s)=s^{2}+m_{\chi_{1}^{0}}^{4}+m_{\chi_{i}^{0}}^{4}-2 s\left(m_{\chi_{1}^{0}}^{2}+m_{\chi_{i}^{0}}^{2}\right)-2 m_{\chi_{1}^{0}}^{2} m_{\chi_{i}^{0}}^{2} .
$$

In order to obtain an approximate expression for the resulting constraints on the parameters, we first neglect $m_{\chi_{1}^{0}}$ everywhere in (3.1). Using numerical values for the gauge couplings, (3.1) simplifies to

$$
\sigma_{Z}\left(e^{+} e^{-} \rightarrow \chi_{1}^{0} \chi_{i}^{0}\right)[\mathrm{pb}] \simeq 4.9 \times 10^{4} \frac{\left(s-m_{\chi_{i}^{0}}^{2}\right)^{2}}{s\left(s-M_{Z}^{2}\right)^{2}}\left(1+\frac{m_{\chi_{i}^{0}}^{2}}{2 s}\right)\left(N_{13} N_{i 3}-N_{14} N_{i 4}\right)^{2}
$$

with $s$ and the masses in $\mathrm{GeV}$. Next we look for approximations for the relevant neutralino mixing parameters $N_{i j}$. For simplification we assume $M_{2}, 2 \kappa s \gg\left|\mu_{\text {eff }}\right|$ such that the wino- and singlino-sectors in the mass matrix $\mathcal{M}_{0}$ in eq. (2.5) decouple. (The wino- and singlino-components of the LSP hardly contribute to the spin-independent cross section.) Assuming, in addition, large $\tan \beta$ such that $v_{d} \ll v_{u}, \mathcal{M}_{0}$ can be diagonalised analytically with the results (we define $u=g_{1} v_{u} / \sqrt{2} \sim 43 \mathrm{GeV}$ and write $\mu \equiv \mu_{\text {eff }}$ )

$$
\begin{array}{lll}
N_{11} \sim \frac{-1}{\sqrt{1+\frac{u^{2}}{\mu^{2}}}}, & N_{13} \sim \frac{-1}{\sqrt{1+\frac{\mu^{2}}{u^{2}}},}, N_{14} \sim 0 \\
N_{21} \sim-N_{31} \sim \frac{1}{\sqrt{2} \sqrt{1+\frac{\mu^{2}}{u^{2}}}}, & N_{23} \sim-N_{33} \sim \frac{-1}{\sqrt{2} \sqrt{1+\frac{u^{2}}{\mu^{2}}}}, \quad N_{24} \sim N_{34} \sim \frac{1}{\sqrt{2}} .
\end{array}
$$

Replacing these expressions into (3.3), using the numerical values for $s$ and $M_{Z}$ in the denominator and, notably, approximating $m_{\chi_{i}^{0}} \sim \mu$, one ends up with

$$
\sigma_{Z}\left(e^{+} e^{-} \rightarrow \chi_{1}^{0} \chi_{i}^{0}\right)[\mathrm{pb}] \simeq 8.3 \times 10^{-7} \frac{\left(s-\mu^{2}\right)^{2} \mu^{2}}{u^{2}+\mu^{2}}\left(1+\frac{\mu^{2}}{2 s}\right)
$$

where $s, \mu$ and $u$ are in $\mathrm{GeV}$. Then the upper OPAL bound on $\sigma_{Z}\left(e^{+} e^{-} \rightarrow \chi_{1}^{0} \chi_{i}^{0}\right)$ of $0.05 \mathrm{pb}$ becomes a lower bound on $|\mu|\left(\equiv \mu_{\mathrm{eff}}\right)$,

$$
\left|\mu_{\mathrm{eff}}\right| \gtrsim 111 \mathrm{GeV}
$$

A somewhat stronger version of the OPAL bound $\left(\sigma_{Z}<0.01 \mathrm{pb}\right)$ is implemented in the default version of NMSSMTools [32, 33]. We replace it by the published value of $0.05 \mathrm{pb}$ [38] for our numerical analysis. From this, without any approximations, we obtain $\left|\mu_{\text {eff }}\right| \gtrsim 120 \mathrm{GeV}$ (varying somewhat with $M_{2}$ and $\tan \beta$ ) for small values of $m_{\chi_{1}^{0}}$ in good 
agreement with the previous estimation. We remark that, within the approximations used in eqs. (3.5), this implies an upper bound on $N_{13}^{2} \lesssim 0.12$.

Next, we consider constraints from the upper bound on the invisible $Z$ decay width, to which the decay $Z \rightarrow \chi_{1}^{0} \chi_{1}^{0}$ would contribute. From [40] we obtain $\Delta \Gamma_{Z}^{\text {inv }} \lesssim 2.0 \mathrm{MeV}$ (a value slightly above the one used in [12], but below the value used in [23]). The expression for the contribution to $\Delta \Gamma_{Z}^{\text {inv }}$ from $\chi_{1}^{0}$ reads

$$
\Delta \Gamma_{Z}^{\mathrm{inv}}=\frac{M_{Z}^{3} G_{F}}{12 \sqrt{2} \pi}\left(N_{13}^{2}-N_{14}^{2}\right)^{2}\left(1-\frac{4 m_{\chi_{1}^{0}}^{2}}{M_{Z}^{2}}\right)^{3 / 2} \sim 0.165 \mathrm{GeV}\left(N_{13}^{2}-N_{14}^{2}\right)^{2}
$$

where the last expression holds for small $m_{\chi_{1}^{0}}$. Then the upper bound on $\Delta \Gamma_{Z}^{\text {inv }}$ implies

$$
\left|N_{13}^{2}-N_{14}^{2}\right|<0.11 \text {. }
$$

For large $\tan \beta$, where $N_{14}^{2} \ll N_{13}^{2}$, this bound on $N_{13}^{2}$ is very similar to the bound obtained above from the OPAL limits. According to the numerical analysis without approximations we find that the constraints on the parameter space from $e^{+} e^{-} \rightarrow \chi_{1}^{0} \chi_{i}^{0}$ are mostly somewhat stronger than those from $\Delta \Gamma_{Z}^{\text {inv }}$; from (2.8) and (2.9) it should be clear, that these constraints are relevant for upper bounds on the spin-independent and spin-dependent LSP-nucleon cross sections.

For the chargino masses we require a lower bound of $103 \mathrm{GeV}$ [41], which implies lower limits on combinations of the parameters $M_{2}$ and $\mu_{\mathrm{eff}}$. In the neutral Higgs sector we apply the various constraints from [42]. Since the lightest CP-even Higgs boson $h$ is mostly Standard-Model-like in our case, these constraints reduce to the well-known bound $m_{h}>114 \mathrm{GeV}$. On the other hand the constraints from B-physics, as described below, will imply charged Higgs masses above $\sim 200 \mathrm{GeV}$, hence additional bounds from direct charged Higgs production are not required.

\subsection{Constraints from B-physics}

Relevant constraints from B-physics originate from bounds on $B R(b \rightarrow s \gamma)=(3.55 \pm 0.24 \pm$ $0.09) \times 10^{-4}[43], \Delta M_{s}=17.77 \pm 0.12 \mathrm{ps}^{-1}[44]$ and $\Delta M_{d}=0.507 \pm 0.005 \mathrm{ps}^{-1}[43]$, and the branching ratios $B R\left(B_{s} \rightarrow \mu^{+} \mu^{-}\right)<5.8 \times 10^{-8}$ [45] (which was recently improved to $<4.3 \times 10^{-8}$ at $95 \%$ C.L. [46] $)$ and $B R\left(B^{+} \rightarrow \tau^{+} \nu_{\tau}\right)<(1.67 \pm 0.39) \times 10^{-4}$ [43]. These constraints are implemented in NMSSMTools as described in [47], to which we refer for the corresponding contributions to these observables in the NMSSM.

It should be noted that charged Higgs boson exchange contributes to $B R(b \rightarrow s \gamma)$ and $B R\left(B^{+} \rightarrow \tau^{+} \nu_{\tau}\right)$, hence the corresponding limits impose lower bounds on $m_{H^{ \pm}}$. Susy diagrams, which depend on parameters like $M_{2}, \mu_{\text {eff }}, M_{\text {squark }}$ and $A_{\text {top }}$ [48], also contribute to $B R(b \rightarrow s \gamma)$. For specific choices of these parameters (notably not too large positive values of $A_{\text {top }}$ ), the charged Higgs boson contribution can be partially cancelled. The charged Higgs boson contribution to $B R\left(B^{+} \rightarrow \tau^{+} \nu_{\tau}\right)$ interferes destructively with the SM $\left(W^{+}\right)$contribution, and the corresponding constraint imposes lower bounds on $m_{H^{ \pm}}$increasing with $\tan \beta$. We resum the Susy large $\tan \beta$ corrections to the charged 
Higgs couplings as in $[48,49]$, which leads to a dependency of the constraints on $\mu_{\text {eff }}$. Bounds from $B \rightarrow D \tau \nu$ could also be relevant [50], but are typically weaker than the other constraints from B-physics at present [51] and not considered here. The lower bounds on $m_{H^{ \pm}}$will be relevant below, since the spin-independent LSP-nucleon cross section (2.8) is maximal for small $m_{H}$ and, as noted above, $m_{H} \sim m_{H^{ \pm}}$.

At large $\tan \beta$, the observables $\Delta M_{s}, \Delta M_{d}$ and $B R\left(B_{s} \rightarrow \mu^{+} \mu^{-}\right)$can receive large contributions from a light CP-odd Higgs boson $A_{1}$ [47] which, in turn, plays an important rôle for the LSP annihilation cross section (2.10) for a small LSP mass $m_{\chi_{1}^{0}}$. Again, additional Susy contributions (box diagrams) exist, leading to a complicated combination of constraints in the parameter space. We find that, for a small LSP mass (light $A_{1}$ ), practically all these observables impose bounds on various corners in the parameter space.

\subsection{Additional constraints}

On the dark matter relic density we impose the $3 \sigma$ WMAP bound [9]

$$
0.091<\Omega h^{2}<0.129
$$

which requires a sufficiently large LSP annihilation rate (2.10).

A light CP-odd Higgs boson $A_{1}$ with a mass below $\sim 9.3 \mathrm{GeV}$ can appear in radiative $\Upsilon \rightarrow A_{1} \gamma$ decays, on which CLEO [52] and BaBar [53, 54] have obtained upper bounds. These can be translated into the parameter space (couplings of $A_{1}$ ) of the NMSSM [55-57] and are implemented, together with constraints from possible $A_{1}-\eta_{b}$ mixing effects [56], in NMSSMTools. We find that these constraints are so strong (imposing, essentially, strong upper bounds on the $A_{1} b \bar{b}$ coupling for $m_{A_{1}} \lesssim 10 \mathrm{GeV}$ ) that it becomes very difficult to obtain a LSP annihilation rate compatible with (3.9) for $m_{\chi_{1}^{0}} \lesssim 2 \mathrm{GeV}$.

Finally we require that the Susy contributions to the anomalous magnetic moment of the muon (see $[58,59]$ for such contributions in the NMSSM) improve the disagreement between the result of the E821 experiment [60] and the Standard Model; as in the MSSM, this implies a positive value for $\mu_{\mathrm{eff}}$.

\section{Results and conclusions}

Before we turn to our results, we discuss the range of parameters used to maximise the direct detection cross sections respecting the experimental constraints above. First, for $\lambda$ we choose a small value $\lambda=0.05$ such that its negative effect on $M_{H^{ \pm}}^{2}$ as in eq. (2.4) remains negligible while a non-zero doublet component of $A_{1}$ is induced by the off-diagonal term in eq. (2.3). A large value for $\kappa=0.55$ makes the singlino (and the singlet-like CP-even Higgs state) heavy such that perturbing mixing effects in these sectors are avoided.

For the Susy breaking squark and slepton masses we use $1 \mathrm{TeV}$ such that sleptons hardly contribute to $e^{+} e^{-} \rightarrow \chi_{1}^{0} \chi_{i}^{0}$. $A_{\text {top }}$ varies from 300 to $650 \mathrm{GeV}$ where $H^{ \pm}$-induced and Susy-induced contributions to $B R(b \rightarrow s \gamma)$ tend to cancel [47]. The Susy breaking gluino and the wino masses are chosen as $M_{3}=350 \mathrm{GeV}$ and $M_{2}=180 \mathrm{GeV}$, respectively. (These parameters appear in the loop-induced flavour changing $A_{1}$-quark vertices [48], 
which should be small in order to allow for a light $A_{1}$ consistent with the constraints from $B R\left(B_{s} \rightarrow \mu^{+} \mu^{-}\right)$.)

Although eq. (2.8) suggests that $\sigma^{\mathrm{SI}}$ is maximised for very large values of $\tan \beta$, the best compromise with B-physics is obtained for reasonable values of $\tan \beta \sim 35-44$. Likewise, eq. (3.5) suggests that $\mu_{\text {eff }}$ should be as small as possible in order to maximise $N_{11} N_{13}$, but we find that for the above values of $M_{2}$ and $\tan \beta$ the bounds from section 3.1 are saturated for $\mu_{\mathrm{eff}} \simeq 128 \mathrm{GeV}$ (the best compromise in parameter space). Eq. (2.8) also suggests that $\sigma^{\mathrm{SI}}$ is maximised for $m_{H}$ as small as possible. However, we recall that $m_{H} \sim M_{A} \sim m_{H^{ \pm}}$ and that $m_{H^{ \pm}}$is bounded from below by several B-physics processes. We choose $M_{A}$ as an input parameter of the NMSSM (instead of $A_{\lambda}$ ) and find the largest values of $\sigma^{\mathrm{SI}}$ for $M_{A} \sim 260-315 \mathrm{GeV}$, implying $m_{H} \sim 205-260 \mathrm{GeV}$ and $m_{H^{ \pm}} \sim 225-280 \mathrm{GeV}$ where the larger values correspond to lower masses of $m_{\chi_{1}^{0}}$ below.

The Susy breaking bino mass term $M_{1}$ determines $m_{\chi_{1}^{0}}$, with $M_{1} \sim 23.5 \mathrm{GeV}$ for $m_{\chi_{1}^{0}} \sim 20 \mathrm{GeV}$ and $M_{1} \sim 3.0 \mathrm{GeV}$ for $m_{\chi_{1}^{0}} \sim 2.0 \mathrm{GeV}$. Finally $A_{\kappa}$ is chosen in the range $A_{\kappa} \sim-14 \ldots-4 \mathrm{GeV}$, which determines $m_{A_{1}}$ such that $\chi_{1}^{0}$ pair annihilation via $A_{1}$ exchange in the s-channel gives the correct relic density in agreement with the WMAP bound in eq. (3.9). Due to the relatively large couplings involved in the $\chi_{1}^{0}$ pair annihilation process, $A_{1}$ must actually be off-shell and hence $m_{A_{1}}$ substantially larger than $2 m_{\chi_{1}^{0}}$; otherwise the relic density is too small. The coupling of the heavy MSSM-like CP-odd Higgs boson $A$ to $\chi_{1}^{0}$ can be twice the coupling of $A_{1}$. However, due to its large mass implied by B-physics constraints, its exchange in the s-channel cannot sufficiently reduce the relic density [17] its contribution to $\sigma_{\text {ann }}$ is always less than the contribution from $A_{1}$ for $m_{\chi_{1}^{0}} \lesssim 20 \mathrm{GeV}$. Although our numerical analysis takes both contributions from $A$ and $A_{1}$ to $\sigma_{\text {ann }}$ into account, the presence of the NMSSM-specific state $A_{1}$ is thus essential for obtaining the correct relic density for this range of $m_{\chi_{1}^{0}}$. In figure 1 we show the result for $m_{A_{1}}$ as function of $m_{\chi_{1}^{0}}$.

For $m_{A_{1}} \lesssim 40 \mathrm{GeV}\left(m_{\chi_{1}^{0}} \lesssim 5 \mathrm{GeV}\right)$ the constraints from $B R\left(B_{s} \rightarrow \mu^{+} \mu^{-}\right)$(where $A_{1}$ appears in the s-channel) become particularly strong and require a somewhat smaller doublet component of $A_{1}$. Denoting its doublet component by $\sin \theta_{A}$, we have $\sin \theta_{A} \sim 0.8$ for $m_{A_{1}} \gtrsim 50 \mathrm{GeV}$, but $\sin \theta_{A} \sim 0.45$ for $m_{A_{1}} \sim 10 \mathrm{GeV}$. We note that for $m_{A_{1}} \lesssim 40 \mathrm{GeV}$ the value of $A_{\kappa}$ has to be chosen within a precision less than $1 \%$ such that the relic density of $\chi_{1}^{0}$ is below the WMAP bound (possibly smaller), but $m_{A_{1}}^{2}>0$; hence this region in the parameter space requires considerable fine tuning. For $m_{A_{1}}<10 \mathrm{GeV}\left(m_{\chi_{1}^{0}} \lesssim 2 \mathrm{GeV}\right)$ the constraints from CLEO and BaBar become so strong that $\sin \theta_{A}$ must be much smaller requiring an even stronger fine tuning of parameters, therefore we will not consider this range of parameters subsequently.

The components of $\chi_{1}^{0}$ (the coefficients $N_{1 i}$, see eq. (2.6)) hardly change in the range $m_{\chi_{1}^{0}}=2-20 \mathrm{GeV}$ considered here, once we maximise the product $N_{11} N_{13}$ in order to maximise $\sigma^{\text {SI }}$. We have

$$
\begin{aligned}
& N_{11} \sim-0.94, N_{12} \sim 0.01 \ldots 0.03, N_{13} \sim-0.32 \ldots-0.34, \\
& N_{14} \sim 0.013 \ldots 0.06, N_{15} \sim 0.001 .
\end{aligned}
$$

The masses of the mostly higgsino-like states $\chi_{2}^{0}$ and $\chi_{3}^{0}$ are $\sim 105$ and $\sim 145 \mathrm{GeV}$, respectively, and hence as stated before, the limits on $\sigma_{Z}\left(e^{+} e^{-} \rightarrow \chi_{1}^{0} \chi_{i}^{0}\right)$ are relevant. 


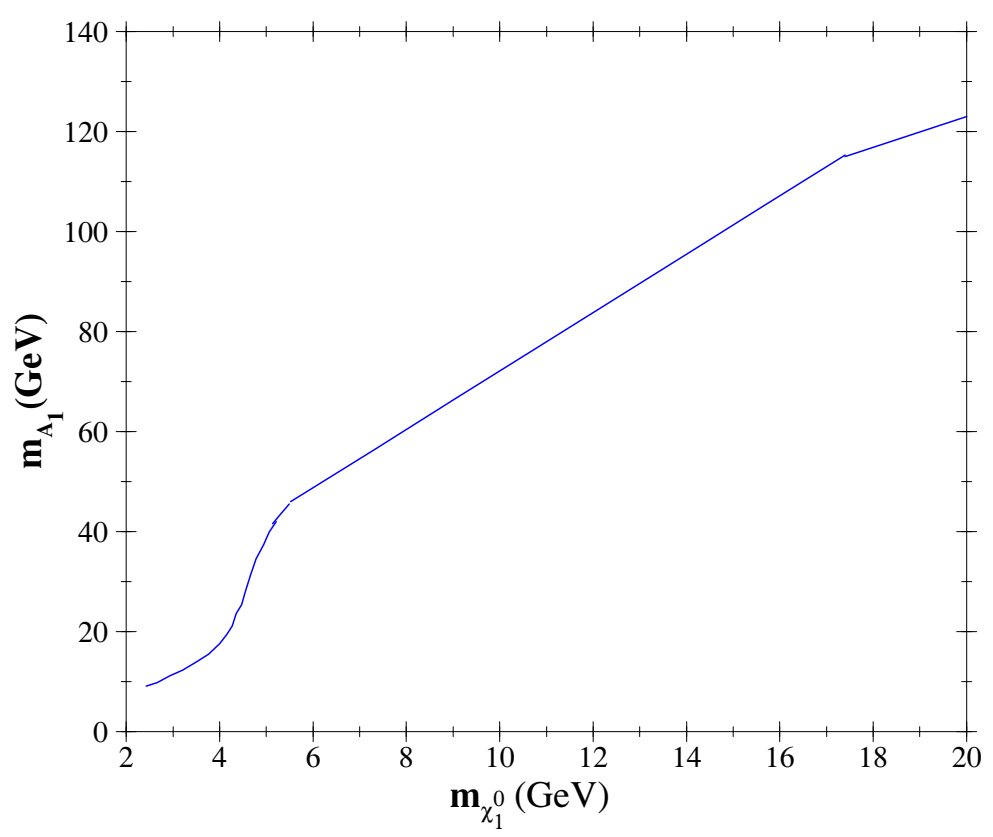

Figure 1. $m_{A_{1}}$ as function of $m_{\chi_{1}^{0}}$ such that the relic density of $\chi_{1}^{0}$ is in agreement with the WMAP bound eq. (3.9).

The scattering rates of $\chi_{1}^{0}$ depend somewhat on astrophysical parameters as the escape velocity $v_{\max }$ and the dark matter density $\rho_{0}$ near the sun and, more importantly, on nuclear form factors (quark matrix elements) as the pion-nucleon sigma term $\sigma_{\pi N}$ and the size of $\mathrm{SU}(3)$ symmetry breaking parametrized by $\sigma_{0}$. (The difference $\sigma_{0}-\sigma_{\pi N}$ is proportional to the strange quark matrix element.) For the astrophysical parameters we use the default values of micrOMEGAs $v_{\max }=544 \mathrm{~km} / \mathrm{s}$ and $\rho_{0}=0.3 \mathrm{GeV} / \mathrm{cm}^{3}$ [36]. The default values in micrOMEGAs for $\sigma_{\pi N}$ and $\sigma_{0}$ are $\sigma_{\pi N}=55 \mathrm{MeV}$ and $\sigma_{0}=35 \mathrm{MeV}$.

The corresponding results for the upper bound on the spin-independent cross section of $\chi_{1}^{0}$ off protons $\sigma_{p}^{\text {SI }}$ in the NMSSM are shown in figure 2 as a function of $m_{\chi_{1}^{0}}$ as a full red line. (The spin-independent cross section off neutrons is nearly the same.) In order to indicate the variation of this upper bound with $\sigma_{\pi N}$ and $\sigma_{0}$, we show a red dashed line as the upper bound on $\sigma_{p}^{\mathrm{SI}}$ for $\sigma_{\pi N}=73 \mathrm{MeV}$ and $\sigma_{0}=30 \mathrm{MeV}$, which would correspond to a larger strange quark matrix element and an increase of $\sigma^{\text {SI }}$ by a factor $\sim 3.3$. This allows to estimate the decrease of the upper bound on $\sigma^{\mathrm{SI}}$, if a smaller value of $\sigma_{\pi N}$ is assumed.

Also shown in figure 2 are the regions compatible with the excesses of events reported by DAMA [1] (without channeling (dark blue) and with channeling (light blue)), CoGeNT [2] (light green) and a fit to the two events observed by CDMS-II [61] (denoted as CDMS09 fit surrounded in dashed green; these events are also compatible with background). Exclusion limits are shown from Xenon10 [5] (violet), Xenon100 [7] (black) and CDMSII $[3,8]$ (magenta, assuming that the two observed events originate from background).

Figure 2 is our main result, which leads to the following conslusions: 


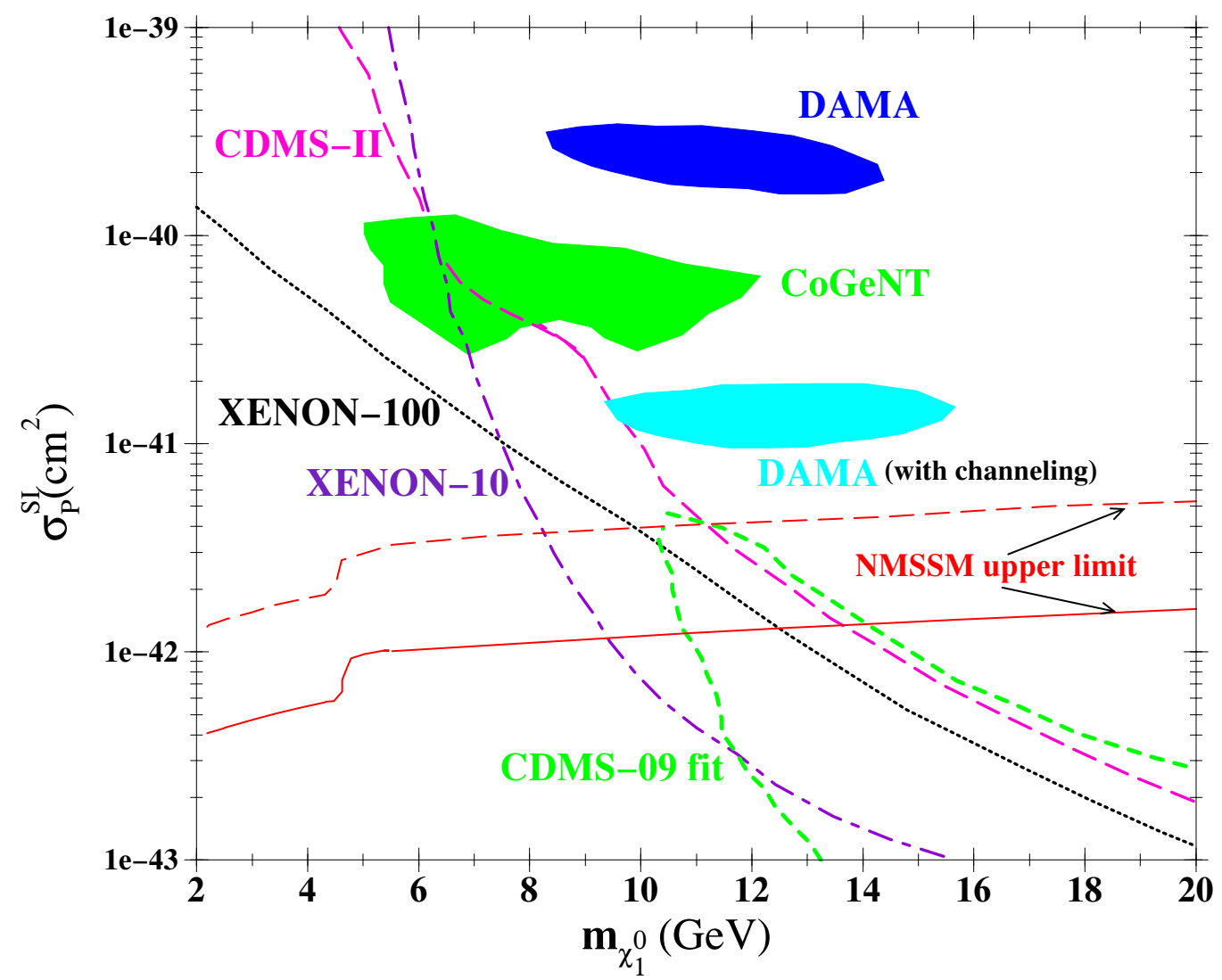

Figure 2. Upper bounds on the spin-independent cross section $\sigma_{p}^{\mathrm{SI}}$ in the NMSSM for default values of the strange quark content of nucleons as a full red line, and an enhanced strange quark content of nucleons as a dashed red line. Also shown are regions compatible with DAMA, CoGeNT and CDMS-II, and limits from Xenon10, Xenon100 and CDMS-II as explained in the text.

- It seems difficult to explain the excesses of events reported by DAMA and CoGeNT within the general NMSSM (without unification constraints on $M_{1}$ ). Hence, as stated in [23], significant modifications of parameters like a larger local dark matter density $\rho_{0}$ would be required to this end. On the other hand, the two events observed by CDMS-II (within the contour denoted as CDMS-09 fit) could be explained in the NMSSM.

- Actual limits of Xenon10, Xenon100 and CDMS-II on spin-independent cross sections of WIMPS in the $2-20 \mathrm{GeV}$ mass range test regions of the parameter space of the NMSSM.

For completeness we have also considered the spin-dependent cross section $\sigma^{\mathrm{SD}}$ in the NMSSM, which is maximal for $\tan \beta \gtrsim 20$ (such that $N_{14}^{2} \ll N_{13}^{2}$ in eq. (2.9)), large values of $M_{A}$ (since $m_{H}$ is irrelevant here), and $\mu_{\text {eff }} \sim 121-129 \mathrm{GeV}$. In figure 3 we show the maximum of the spin-dependent cross section off protons $\sigma_{p}^{\mathrm{SD}}$ for the same range of $m_{\chi_{1}^{0}}=2-20 \mathrm{GeV}$. Note that $\sigma^{\mathrm{SD}}$ originates from $Z$-exchange, hence the spin-dependent 


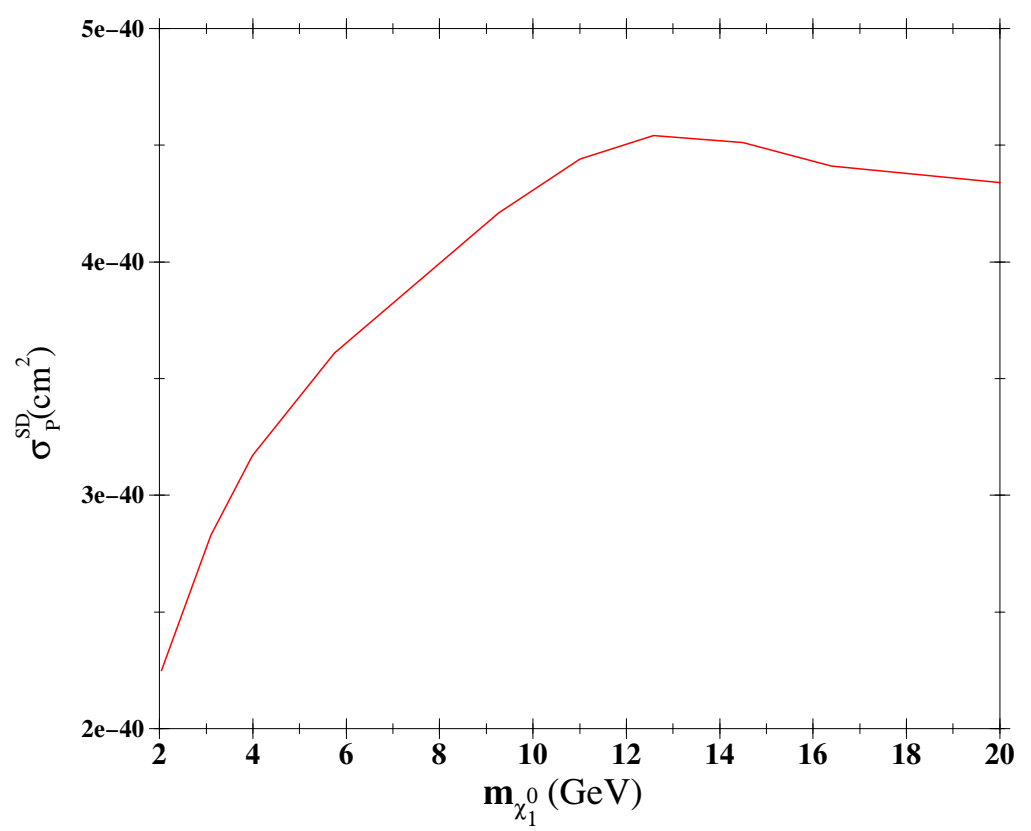

Figure 3. Upper bounds on the spin-dependent cross section $\sigma_{p}^{\mathrm{SD}}$ in the NMSSM.

cross section off neutrons $\sigma_{n}^{\mathrm{SD}}$ is given by $\sigma_{n}^{\mathrm{SD}} \simeq 0.78 \times \sigma_{p}^{\mathrm{SD}}$. The actual experimental upper limits on $\sigma^{\mathrm{SD}}$ are one to two orders of magnitude larger [62] than the upper bounds in the NMSSM and not shown in figure 3.

To conclude, we have performed a detailed analysis of the parameter space of the NMSSM for general values of $M_{1}$, which allows for WIMP masses in the $2-20 \mathrm{GeV}$ range. In contrast to the MSSM, light bino-like WIMPs can have a relic density compatible with WMAP constraints due to a light NMSSM-specific CP-odd Higgs state which can be exchanged in the s-channel. Due to reported excesses of events compatible with WIMP masses below $20 \mathrm{GeV}$, this region is of particular interest.

We have studied in detail the constraints on this region of the parameter space of the NMSSM from LEP and B-physics, and the regions of parameter space which give rise to maximal direct detection cross sections while not contradicting experimental limits. The resulting upper bounds on $\sigma^{\mathrm{SI}} \lesssim 10^{-42} \mathrm{~cm}^{2}=10^{-6} \mathrm{pb}$ make it difficult to explain the excesses of events reported by DAMA and CoGeNT within the NMSSM for small values of $M_{1}$. On the other hand, the two events observed by CDMS-II could be explained in the NMSSM.

Notably the Xenon10 limits [5] on $\sigma^{\mathrm{SI}}$ for WIMP masses below $20 \mathrm{GeV}$ start to test corresponding regions of the NMSSM parameter space. Future results from Xenon100 could confirm the presence of a light WIMP compatible with the NMSSM, or impose further constraints on its parameter space.

\section{Acknowledgments}

We thank A. Goudelis and Y. Mambrini for discussions. D.D. acknowledges support from the Groupement d'Intérêt Scientifique P2I. 
Open Access. This article is distributed under the terms of the Creative Commons Attribution Noncommercial License which permits any noncommercial use, distribution, and reproduction in any medium, provided the original author(s) and source are credited.

\section{References}

[1] DAMA collaboration, R. Bernabei et al., First results from DAMA/LIBRA and the combined results with DAMA/NaI, Eur. Phys. J. C 56 (2008) 333 [arXiv:0804.2741] [SPIRES].

[2] CoGeNT collaboration, C.E. Aalseth et al., Results from a Search for Light-Mass Dark Matter with a P-type Point Contact Germanium Detector, arXiv: 1002.4703 [SPIRES].

[3] The CDMS-II collaboration, Z. Ahmed et al., Dark Matter Search Results from the CDMS II Experiment, Science 327 (2010) 1619 [arXiv:0912.3592] [SPIRES].

[4] G. Angloher et al., Limits on WIMP dark matter using sapphire cryogenic detectors, Astropart. Phys. 18 (2002) 43 [SPIRES].

[5] XENON collaboration, J. Angle et al., First Results from the XENON10 Dark Matter Experiment at the Gran Sasso National Laboratory, Phys. Rev. Lett. 100 (2008) 021303 [arXiv:0706.0039] [SPIRES].

[6] TEXONO collaboration, S.T. Lin et al., New limits on spin-independent and spin-dependent couplings of low-mass WIMP dark matter with a germanium detector at a threshold of 220 eV, Phys. Rev. D 79 (2009) 061101 [arXiv:0712.1645] [SPIRES].

[7] XENON100 collaboration, E. Aprile et al., First Dark Matter Results from the XENON100 Experiment, Phys. Rev. Lett. 105 (2010) 131302 [arXiv: 1005.0380] [SPIRES].

[8] CDMS collaboration, D.S. Akerib et al., Limits on spin-independent WIMP nucleon interactions from the two-tower run of the Cryogenic Dark Matter Search, Phys. Rev. Lett. 96 (2006) 011302 [astro-ph/0509259] [SPIRES].

[9] WMAP collaboration, E. Komatsu et al., Five-Year Wilkinson Microwave Anisotropy Probe (WMAP) Observations: Cosmological Interpretation, Astrophys. J. Suppl. 180 (2009) 330 [arXiv: 0803.0547] [SPIRES].

[10] D. Hooper and T. Plehn, Supersymmetric dark matter: How light can the LSP be?, Phys. Lett. B 562 (2003) 18 [hep-ph/0212226] [SPIRES].

[11] G. Bélanger, F. Boudjema, A. Pukhov and S. Rosier-Lees, A lower limit on the neutralino mass in the MSSM with non- universal gaugino masses. ((T)) ((U)), hep-ph/0212227 [SPIRES].

[12] H.K. Dreiner et al., Mass Bounds on a Very Light Neutralino, Eur. Phys. J. C 62 (2009) 547 [arXiv: 0901.3485] [SPIRES].

[13] A. Bottino, N. Fornengo and S. Scopel, Light relic neutralinos, Phys. Rev. D 67 (2003) 063519 [hep-ph/0212379] [SPIRES].

[14] A. Bottino, F. Donato, N. Fornengo and S. Scopel, Light neutralinos and WIMP direct searches, Phys. Rev. D 69 (2004) 037302 [hep-ph/0307303] [SPIRES].

[15] G. Bélanger, F. Boudjema, A. Cottrant, A. Pukhov and S. Rosier-Lees, Lower limit on the neutralino mass in the general MSSM, JHEP 03 (2004) 012 [hep-ph/0310037] [SPIRES]. 
[16] A. Bottino, F. Donato, N. Fornengo and S. Scopel, Interpreting the recent results on direct search for dark matter particles in terms of relic neutralino, Phys. Rev. D 78 (2008) 083520 [arXiv:0806.4099] [SPIRES].

[17] D. Feldman, Z. Liu and P. Nath, Low Mass Neutralino Dark Matter in the MSSM with Constraints from $B_{s} \rightarrow \mu^{+} \mu^{-}$and Higgs Search Limits, Phys. Rev. D 81 (2010) 117701 [arXiv: 1003.0437] [SPIRES].

[18] E. Kuflik, A. Pierce and K.M. Zurek, Light Neutralinos with Large Scattering Cross Sections in the Minimal Supersymmetric Standard Model, Phys. Rev. D 81 (2010) 111701 [arXiv: 1003.0682] [SPIRES].

[19] M. Maniatis, The Next-to-Minimal Supersymmetric extension of the Standard Model reviewed, Int. J. Mod. Phys. A 25 (2010) 3505 [arXiv:0906.0777] [SPIRES].

[20] U. Ellwanger, C. Hugonie and A.M. Teixeira, The Next-to-Minimal Supersymmetric Standard Model, to appear in Phys. Rept, arXiv:0910.1785 [SPIRES].

[21] B. McElrath, Invisible quarkonium decays as a sensitive probe of dark matter, Phys. Rev. D 72 (2005) 103508 [hep-ph/0506151] [SPIRES].

[22] V. Barger, P. Langacker and H.-S. Lee, Lightest neutralino in extensions of the MSSM, Phys. Lett. B 630 (2005) 85 [hep-ph/0508027] [SPIRES].

[23] J.F. Gunion, D. Hooper and B. McElrath, Light neutralino dark matter in the NMSSM, Phys. Rev. D 73 (2006) 015011 [hep-ph/0509024] [SPIRES].

[24] J. Cao, H.E. Logan and J.M. Yang, Experimental constraints on NMSSM and implications on its phenomenology, Phys. Rev. D 79 (2009) 091701 [arXiv:0901.1437] [SPIRES].

[25] D.G. Cerdeno, C. Hugonie, D.E. Lopez-Fogliani, C. Muñoz and A.M. Teixeira, Theoretical predictions for the direct detection of neutralino dark matter in the NMSSM, JHEP 12 (2004) 048 [hep-ph/0408102] [SPIRES].

[26] D.G. Cerdeno, E. Gabrielli, D.E. Lopez-Fogliani, C. Muñoz and A.M. Teixeira, Phenomenological viability of neutralino dark matter in the NMSSM, JCAP 06 (2007) 008 [hep-ph/0701271] [SPIRES].

[27] V. Barger et al., Recoil detection of the lightest neutralino in MSSM singlet extensions, Phys. Rev. D 75 (2007) 115002 [hep-ph/0702036] [SPIRES].

[28] G. Bélanger, C. Hugonie and A. Pukhov, Precision measurements, dark matter direct detection and LHC Higgs searches in a constrained NMSSM, JCAP 01 (2009) 023 [arXiv: 0811.3224] [SPIRES].

[29] N. Yokozaki, Electroweak Symmetry Breaking and Singlino Dark Matter with Deflected Anomaly Mediation, JHEP 05 (2009) 095 [arXiv: 0903.2632] [SPIRES].

[30] J. Cao, K.-i. Hikasa, W. Wang, J.M. Yang and L.-X. Yu, SUSY dark matter in light of CDMS II results: a comparative study for different models, JHEP 07 (2010) 044 [arXiv: 1005.0761] [SPIRES].

[31] S. Demidov and O. Suvorova, Annihilation of NMSSM neutralinos in the Sun and neutrino telescope limits, JCAP 06 (2010) 018 [arXiv:1006.0872] [SPIRES].

[32] U. Ellwanger, J.F. Gunion and C. Hugonie, NMHDECAY: A Fortran code for the Higgs masses, couplings and decay widths in the NMSSM, JHEP 02 (2005) 066 [hep-ph/0406215] [SPIRES]. 
[33] U. Ellwanger and C. Hugonie, NMHDECAY 2.0: An Updated program for sparticle masses, Higgs masses, couplings and decay widths in the NMSSM,

Comput. Phys. Commun. 175 (2006) 290 [hep-ph/0508022] [SPIRES].

[34] G. Bélanger, F. Boudjema, C. Hugonie, A. Pukhov and A. Semenov, Relic density of dark matter in the NMSSM, JCAP 09 (2005) 001 [hep-ph/0505142] [SPIRES].

[35] G. Bélanger, F. Boudjema, A. Pukhov and A. Semenov, MicrOMEGAs2.0: A program to calculate the relic density of dark matter in a generic model, Comput. Phys. Commun. 176 (2007) 367 [hep-ph/0607059] [SPIRES].

[36] G. Bélanger, F. Boudjema, A. Pukhov and A. Semenov, Dark matter direct detection rate in a generic model with MicrOMEGAs2.1, Comput. Phys. Commun. 180 (2009) 747 [arXiv: 0803.2360] [SPIRES].

[37] DELPHI collaboration, J. Abdallah et al., Searches for supersymmetric particles in $e^{+} e^{-}$ collisions up to $208 \mathrm{GeV}$ and interpretation of the results within the MSSM,

Eur. Phys. J. C 31 (2003) 421 [hep-ex/0311019] [SPIRES].

[38] OPAL collaboration, G. Abbiendi et al., Search for chargino and neutralino production at $\sqrt{s}=192 \mathrm{GeV}$ to $209 \mathrm{GeV}$ at LEP, Eur. Phys. J. C 35 (2004) 1 [hep-ex/0401026] [SPIRES].

[39] F. Franke and H. Fraas, Production and decay of neutralinos in the next-to-minimal supersymmetric standard model, Z. Phys. C 72 (1996) 309 [hep-ph/9511275] [SPIRES].

[40] ALEPH, DELPHI, L3, OPAL, SLD, LEP Electroweak Working Group, SLD ELECTROWEAK, HEAVY FLAVOUR GROUPS collaborations, Precision electroweak measurements on the $Z$ resonance, Phys. Rept. 427 (2006) 257 [hep-ex/0509008] [SPIRES].

[41] ALEPH, DELPHI, L3, OPAL collaborations, LEP2 Susy Working Group, Combined LEP Chargino Results, up to $208 \mathrm{GeV}$ for large mo, http://lepsusy.web.cern.ch/lepsusy/www/inos_moriond01/charginos_pub.html.

[42] ALEPH, DELPHI, L3, OPAL collaborations, LEP Working Group "For HigGS Boson Searches", S. Schael et al., Search for neutral MSSM Higgs bosons at LEP, Eur. Phys. J. C 47 (2006) 547 [hep-ex/0602042] [SPIRES].

[43] Heavy Flavor Averaging Group collaboration, E. Barberio et al., Averages of b-hadron and c-hadron Properties at the End of 2007, arXiv:0808.1297 [SPIRES].

[44] CDF collaboration, A. Abulencia et al., Observation of $B / s 0$ anti-B/s0 oscillations, Phys. Rev. Lett. 97 (2006) 242003 [hep-ex/0609040] [SPIRES].

[45] CDF collaboration, T. Aaltonen et al., Search for $B_{s}^{0} \rightarrow \mu^{+} \mu^{-}$and $B_{d}^{0} \rightarrow \mu^{+} \mu^{-}$decays with $2 \mathrm{fb}^{-1}$ of $p \bar{p}$ collisions, Phys. Rev. Lett. 100 (2008) 101802 [arXiv: 0712.1708] [SPIRES].

[46] CDF, D0 collaborations, M.J. Morello, Flavor Changing Neutral Current at the Tevatron, arXiv:0912.2446 [SPIRES].

[47] F. Domingo and U. Ellwanger, Updated Constraints from B Physics on the MSSM and the NMSSM, JHEP 12 (2007) 090 [arXiv:0710.3714] [SPIRES].

[48] A.J. Buras, P.H. Chankowski, J. Rosiek and L. Slawianowska, $\Delta M_{d, s}, B^{0} d, s \rightarrow \mu^{+} \mu^{-}$and $B \rightarrow X_{s} \gamma$ in supersymmetry at large $\tan \beta$, Nucl. Phys. B 659 (2003) 3 [hep-ph/0210145] [SPIRES]. 
[49] A.G. Akeroyd and S. Recksiegel, The effect of $H^{ \pm}$on $B^{ \pm} \rightarrow \tau^{ \pm} \nu_{\tau}$ and $B^{ \pm} \rightarrow \mu^{ \pm} \nu_{\mu}$, J. Phys. G 29 (2003) 2311 [hep-ph/0306037] [SPIRES].

[50] U. Nierste, S. Trine and S. Westhoff, Charged-Higgs effects in a new $B \rightarrow D \tau \nu$ differential decay distribution, Phys. Rev. D 78 (2008) 015006 [arXiv:0801.4938] [SPIRES].

[51] B. Dudley and C. Kolda, Constraining the Charged Higgs Mass in the MSSM: A LowEnergy Approach, arXiv:0901.3337 [SPIRES].

[52] CLEO collaboration, W. Love et al., Search for Very Light CP-Odd Higgs Boson in Radiative Decays of $\Upsilon(S-1)$, Phys. Rev. Lett. 101 (2008) 151802 [arXiv:0807.1427] [SPIRES].

[53] BABAR collaboration, B. Aubert et al., Search for Dimuon Decays of a Light Scalar Boson in Radiative Transitions $\Upsilon \rightarrow \gamma A_{0}$, Phys. Rev. Lett. 103 (2009) 081803 [arXiv:0905.4539] [SPIRES].

[54] BABAR collaboration, B. Aubert et al., Search for a low-mass Higgs boson in $Y(3 S) \rightarrow \gamma A^{0}, A^{0} \rightarrow \tau^{+} \tau^{-}$at BABAR, Phys. Rev. Lett. 103 (2009) 181801 [arXiv:0906.2219] [SPIRES].

[55] R. Dermisek, J.F. Gunion and B. McElrath, Probing NMSSM scenarios with minimal fine-tuning by searching for decays of the $\Upsilon$ to a light CP-odd Higgs boson, Phys. Rev. D 76 (2007) 051105 [hep-ph/0612031] [SPIRES].

[56] F. Domingo, U. Ellwanger, E. Fullana, C. Hugonie and M.-A. Sanchis-Lozano, Radiative $\Upsilon$ decays and a light pseudoscalar Higgs in the NMSSM, JHEP 01 (2009) 061 [arXiv: 0810.4736] [SPIRES].

[57] R. Dermisek and J.F. Gunion, New constraints on a light CP-odd Higgs boson and related NMSSM Ideal Higgs Scenarios, Phys. Rev. D 81 (2010) 075003 [arXiv:1002.1971] [SPIRES].

[58] J.F. Gunion, A light CP-odd Higgs boson and the muon anomalous magnetic moment, JHEP 08 (2009) 032 [arXiv:0808.2509] [SPIRES].

[59] F. Domingo and U. Ellwanger, Constraints from the Muon g-2 on the Parameter Space of the NMSSM, JHEP 07 (2008) 079 [arXiv:0806. 0733] [SPIRES].

[60] Muon G-2 collaboration, G.W. Bennett et al., Final report of the muon E821 anomalous magnetic moment measurement at BNL, Phys. Rev. D 73 (2006) 072003 [hep-ex/0602035] [SPIRES].

[61] J. Kopp, T. Schwetz and J. Zupan, Global interpretation of direct Dark Matter searches after CDMS-II results, JCAP 02 (2010) 014 [arXiv:0912.4264] [SPIRES].

[62] C. Savage, G. Gelmini, P. Gondolo and K. Freese, Compatibility of DAMA/LIBRA dark matter detection with other searches, JCAP 04 (2009) 010 [arXiv:0808.3607] [SPIRES]. 